\title{
Stark-Chirped Rapid Adiabatic Passage in a Multilevel Atom
}

\author{
M. RAdOnJiĆ* AND B.M. JelenkoviĆ \\ Institute of Physics, University of Belgrade, Pregrevica 118, 11080 Belgrade, Belgrade, Serbia
}

\begin{abstract}
We generalize Stark-chirped rapid adiabatic passage to the case of a multilevel atom. The atom is assumed to have two ground state levels $g$ and $f$ and an excited level $e$. The adiabatic passage is carried out by resonantly driving the $g-e$ and $f-e$ transitions with time-dependent pump and Stokes fields in the presence of strong far-off-resonant Stark field. Our formalism determines the states that participate in population transfer and is applicable to systems with arbitrary numbers of degenerate states in each level and arbitrary couplings of the $g-e$ and $f-e$ transitions. We give an application to adiabatic passage in $\mathrm{Rb}$ atom.
\end{abstract}

PACS numbers: 32.80.Qk, 32.80.Xx, 33.80.Be

\section{Introduction}

In a recently proposed technique of Stark-chirped rapid adiabatic passage (SCRAP), the population transfer among two atomic states is efficiently driven by the pump laser, while an intense, far off-resonant Stark laser modifies the transition probability between the two states by Stark-shifting their energies [1]. In this process the pump laser frequency is nearly resonant to the atomic transition so that ac Stark shift produces two separate crossings of diabatic energies during the Stark laser pulse. The essential element is that the pump Rabi frequency has to be appreciable only at one of the level crossings. It has been shown that the proper pump pulse intensity, duration, and partial overlap with the Stark pulse produce complete population transfer from one state to another [2]. Recently, SCRAP technique was extended for complete population transfer amongst three states. In the technique all three states have to be coupled simultaneously using three laser pulses. Near resonant Stokes laser pulse is applied in addition to the pump and the Stark laser pulses [3]. The population transfer is efficient if the population of an intermediate state is minimized by the proper laser pulse timing and by adiabatic evolution of the states. This technique was experimentally used for preparation of population inversion in NO molecules $[4,5]$.

In this work we present the SCRAP technique extended to a multilevel system consisting of three degenerate-level manifolds with arbitrary number of sublevels. The approach relies on the decomposition of the Hilbert space of the system into minimal invariant subspaces to which the evolution of the system is restricted [6]. The method is applied to the adiabatic passage among three hyperfine levels in the Rb atom.

\footnotetext{
* corresponding author; e-mail: milanr@phy.bg.ac.rs
}

\section{SCRAP in a three-level atom}

In this section we introduce the notation and set the stage for a general formalism. Consider SCRAP population transfer among three degenerate-level manifolds (ground $g$, excited $e$, and final $f$ ), having energies $E_{g(e, f)}$. The transitions $g-e$ and $f-e$ are driven by classical field pump and Stokes pulses, respectively. Strong off-resonant Stark field pulse is used to introduce dynamic Stark detunings. Let $\mathcal{G}=\left\{\left|g_{i}\right\rangle \mid i=1, \ldots, n_{g}\right\}$, $\mathcal{F}=\left\{\left|f_{j}\right\rangle \mid j=1, \ldots, n_{f}\right\}$, and $\mathcal{E}=\left\{\left|e_{k}\right\rangle \mid k=1, \ldots, n_{e}\right\}$ denote the bases of Hilbert spaces for manifolds $g, f$, and $e$, respectively, consisting of bare atomic states. In the same manner, the state $|\Psi(t)\rangle$ of the system is now represented in basis $\mathcal{E} \cup \mathcal{G} \cup \mathcal{F}$ by the vector $\boldsymbol{C}(t)$ incorporating explicit phases taken from carrier frequency of the pump and Stokes pulses, $\omega_{\mathrm{p}}$ and $\omega_{\mathrm{S}}$. We are concerned with coherent excitation so we will describe the dynamics by the time-dependent Schrödinger equation. In the rotating-wave picture and using rotating-wave approximation (RWA) we get the time-dependent Schrödinger equation for $\boldsymbol{C}(t)$

$$
\mathrm{i} \hbar \frac{\mathrm{d}}{\mathrm{d} t} \boldsymbol{C}(t)=\boldsymbol{H}(t) \boldsymbol{C}(t) .
$$

Matrix representation $\boldsymbol{H}(t)$ of the system Hamiltonian has the form

$$
\begin{aligned}
& \boldsymbol{H}(t)=\hbar \\
& \times\left[\begin{array}{ccc}
\Delta_{e}+\mathcal{S}(t) \boldsymbol{S}_{\mathrm{e}} & (1 / 2) \Omega_{\mathrm{p}}(t) \boldsymbol{V}_{g}^{\dagger} & (1 / 2) \Omega_{\mathrm{S}}(t) \boldsymbol{V}_{f}^{\dagger} \\
(1 / 2) \Omega_{\mathrm{p}}(t) \boldsymbol{V}_{g} & \mathcal{S}(t) \boldsymbol{S}_{\mathrm{g}} & \mathbf{0} \\
(1 / 2) \Omega_{\mathrm{S}}(t) \boldsymbol{V}_{f} & \mathbf{0} & \Delta_{f}+\mathcal{S}(t) \boldsymbol{S}_{f}
\end{array}\right],
\end{aligned}
$$

where $\Omega_{\mathrm{p}}(t)$ and $\Omega_{\mathrm{S}}(t)$ are Rabi frequencies of the pump and Stokes field, respectively, and $\boldsymbol{V}_{g}\left(\boldsymbol{V}_{f}\right)$ is the matrix representation of lowering operator that connects the states in manifold $e$ to the states in manifold $g(f)$. The zeros $\mathbf{0}$ denote null rectangular matrices of appropriate dimensions. The diagonal matrices $\boldsymbol{\Delta}_{e}$ and $\boldsymbol{\Delta}_{f}$ describe static detunings and can be represented as $\boldsymbol{\Delta}_{e}=\Delta_{e} \mathbf{1}_{n_{e}}$ and $\boldsymbol{\Delta}_{f}=\Delta_{f} \mathbf{1}_{n_{f}}$, where common static detunings $\Delta_{e}$ 
and $\Delta_{f}$ for one-photon transitions are given by

$$
\begin{aligned}
& \Delta_{e}=\left(E_{e}-E_{g}\right) / \hbar-\omega_{\mathrm{p}}, \\
& \Delta_{f}=\left(E_{f}-E_{g}\right) / \hbar+\omega_{\mathrm{S}}-\omega_{\mathrm{p}} .
\end{aligned}
$$

The matrices $\boldsymbol{S}_{\mathrm{e}}, \boldsymbol{S}_{\mathrm{g}}$ and $\boldsymbol{S}_{f}$ correspond to the Stark shift operators of the states in manifolds $e, g$ and $f$, respectively. Their diagonal elements are proportional to the Stark shifts of the sublevels. All Stark shifts share the same time dependence, expressed by $\mathcal{S}(t)$, that arises from the laser Stark field variation in time. The quantity $\mathcal{S}(t)$ is proportional to the Stark pulse envelope and may coincide with some suitably chosen referent Stark shift. The structure of the RWA Hamiltonian of Eq. (2) is similar to that of the conventional three-state SCRAP with single elements replaced by the matrices.

The key idea of our approach is to simplify the analysis of a complex multilevel system by subdivision into a set of smaller independently evolving subsystems. It is necessary to find the decomposition of the state space into a set of minimal-sized subspaces to which the evolution is restricted. These subspaces correspond to minimal-sized invariant subspaces (hereafter, invariant subspaces) of the Hamiltonian $\boldsymbol{H}(t)$. Therefore, the analysis of a multilevel system is performed essentially by identifying the Hamiltonian invariant subspaces that depend substantially on couplings of the transitions and sublevel Stark shifts. The details of the formalism are given elsewhere [6].

Here we analyze SCRAP in ${ }^{87} \mathrm{Rb}$ from the ground hyperfine level $5 S_{1 / 2}, F_{g}=2$ to the final level $5 S_{1 / 2}, F_{f}=1$ via the excited level $5 P_{1 / 2}, F_{e}=1$. Transitions $g-e$ and $f-e$ are driven by classical fields, pump and Stokes respectively, with corresponding atomic lowering operators given by

$$
\hat{V}_{g}=\widehat{\boldsymbol{V}}_{g} \cdot \hat{\epsilon}_{\mathrm{p}}, \quad \hat{V}_{f}=\widehat{\boldsymbol{V}}_{f} \cdot \hat{\epsilon}_{\mathrm{S}},
$$

where $\hat{\epsilon}_{\mathrm{p}}$ and $\hat{\epsilon}_{\mathrm{S}}$ are the polarizations of the pump and Stokes field. We choose the coordinate system such that the fields propagate along the $z$ axis, and define a basis of Zeeman states relative to this quantization axis. Bases of Hilbert spaces for manifolds $e, f$ and $g$ are

$$
\begin{aligned}
& \mathcal{E}=\left\{|1,-1\rangle_{e},|1,0\rangle_{e},|1,1\rangle_{e}\right\}, \\
& \mathcal{F}=\left\{|1,-1\rangle_{f},|1,0\rangle_{f},|1,1\rangle_{f}\right\}, \\
& \mathcal{G}=\left\{|2,-2\rangle_{g},|2,-1\rangle_{g},|2,0\rangle_{g},|2,1\rangle_{g},|2,2\rangle_{g}\right\} .
\end{aligned}
$$

We assume that the Stark field is linearly polarized, so the degeneracy of ground levels is preserved. Excited hyperfine sublevels gain both scalar and tensor shifts. To assure necessary conditions for adiabatic connection between ground and final level [3], we choose pump and Stokes carrier frequencies such that $\Delta_{f}<0$ and $\Delta_{e}>0$, and take Stark field frequency so that the Stark shifts of the $e(g$ and $f$ ) sublevels are negative (positive). Off-diagonal elements of the Stark shift operators are neglected for simplicity. Sublevel Stark shifts have the form

$$
\begin{aligned}
& \boldsymbol{S}_{\mathrm{g}}=s_{g} \operatorname{diag}\{\underbrace{1, \ldots, 1}_{2 F_{g}+1}\}, \quad \boldsymbol{S}_{f}=s_{f} \operatorname{diag}\{\underbrace{1, \ldots, 1}_{2 F_{f}+1}\}, \\
& \boldsymbol{S}_{\mathrm{e}}=\operatorname{diag}\left\{-\left(1+s_{e} m_{e}^{2} / F_{e}^{2}\right) \mid m_{e}=-F_{e}, \ldots, F_{e}\right\},
\end{aligned}
$$

where $s_{g}, s_{f}$ and $s_{e}$ are constants chosen arbitrarily in the present example. $\mathcal{S}(t)$ is taken equal to the absolute value of the Stark shift of the excited sublevel $|1,0\rangle_{e}$. For numerical results we assume Gaussian shapes for all pulses, and take the pump and Stokes Rabi frequencies to have identical peak values $\Omega_{0}$, obtaining

$$
\begin{aligned}
& \Omega_{\mathrm{p}}(t)=\Omega_{0} \mathrm{e}^{-\left(t-\tau_{\mathrm{p}}\right)^{2} / T_{\mathrm{p}}^{2}}, \quad \Omega_{\mathrm{S}}(t)=\Omega_{0} \mathrm{e}^{-\left(t-\tau_{\mathrm{S}}\right)^{2} / T_{\mathrm{S}}^{2}}, \\
& \mathcal{S}(t)=S_{0} \mathrm{e}^{-t^{2} / T^{2}} .
\end{aligned}
$$

We will also take equal pump and Stokes durations, $T_{\mathrm{p}}=T_{\mathrm{S}}$ and $T=2 T_{\mathrm{p}}$. Stark field peak value is taken large enough to assure necessary diabatic energy crossings. The timings $\tau_{\mathrm{p}}$ and $\tau_{\mathrm{S}}$ of pulses are chosen to correspond to appropriate first crossings of diabatic energies of ground and final level with diabatic energy of excited sublevel $|1,0\rangle_{e}$, in the "counterintuitive" order [3]. The polarizations of the pump and Stokes field are both chosen to be linear along $x$ axis, so the matrix representations of lowering operators are

$$
\begin{aligned}
& \boldsymbol{V}_{g}=\left[\begin{array}{ccc}
1 / 2 & 0 & 0 \\
0 & 1 / \sqrt{8} & 0 \\
1 / \sqrt{24} & 0 & 1 / \sqrt{24} \\
0 & 1 / \sqrt{8} & 0 \\
0 & 0 & 1 / 2
\end{array}\right], \\
& \boldsymbol{V}_{f}=\left[\begin{array}{ccc}
0 & -1 / \sqrt{24} & 0 \\
1 / \sqrt{24} & 0 & -1 / \sqrt{24} \\
0 & 1 / \sqrt{24} & 0
\end{array}\right] .
\end{aligned}
$$

Let $\mathcal{H}_{g e}^{\mathrm{d}}=\operatorname{ker} \boldsymbol{V}_{g}^{\dagger}\left(\mathcal{H}_{f e}^{\mathrm{d}}=\operatorname{ker} \boldsymbol{V}_{f}^{\dagger}\right)$ be the subspace of states in manifold $g(f)$ that are dark to the transition $g \rightarrow e(f \rightarrow e)$, and let $\mathcal{H}_{\text {ef }}^{\mathrm{d}}=\operatorname{ker} \boldsymbol{V}_{f}$ be the subspace of states in manifold $e$ that are dark to the transition. We obtain the following dark subspaces:

$$
\begin{gathered}
\mathcal{H}_{g e}^{\mathrm{d}}=\operatorname{span}\left\{1 / \sqrt{8}|2,-2\rangle_{g}+\sqrt{3} / 2|2,0\rangle_{g}\right. \\
\left.\quad+1 / \sqrt{8}|2,2\rangle_{g}, 1 / \sqrt{2}|2,-1\rangle_{g}+1 / \sqrt{2}|2,1\rangle_{g}\right\}, \\
\mathcal{H}_{f e}^{\mathrm{d}}=\operatorname{span}\left\{1 / \sqrt{2}|1,-1\rangle_{f}-1 / \sqrt{2}|1,1\rangle_{f}\right\}, \\
\mathcal{H}_{\text {ef }}^{\mathrm{d}}=\operatorname{span}\left\{1 / \sqrt{2}|1,-1\rangle_{e}-1 / \sqrt{2}|1,1\rangle_{e}\right\} .
\end{gathered}
$$

There are three common invariant subspaces for $\boldsymbol{V}_{g}^{\dagger} \boldsymbol{V}_{g}, \boldsymbol{V}_{f}^{\dagger} \boldsymbol{V}_{f}$ and $\boldsymbol{S}_{\mathrm{e}}$

$$
\begin{aligned}
& \mathcal{H}_{e, 1}^{\text {inv }}=\operatorname{span}\left\{\frac{1}{\sqrt{2}}|1,-1\rangle_{e}-\frac{1}{\sqrt{2}}|1,1\rangle_{e}\right\}, \\
& \mathcal{H}_{e, 2}^{\text {inv }}=\operatorname{span}\left\{\frac{1}{\sqrt{2}}|1,-1\rangle_{e}+\frac{1}{\sqrt{2}}|1,1\rangle_{e}\right\}, \\
& \mathcal{H}_{e, 3}^{\text {inv }}=\operatorname{span}\left\{|1,0\rangle_{e}\right\},
\end{aligned}
$$

five invariant subspaces for $\boldsymbol{V}_{g} \boldsymbol{V}_{g}^{\dagger}$ and $\boldsymbol{S}_{\mathrm{g}}$ 


$$
\begin{aligned}
& \mathcal{H}_{g, 1}^{\text {inv }}=\operatorname{span}\left\{\sqrt{\frac{3}{8}}|2,-2\rangle_{g}-\frac{1}{2}|2,0\rangle_{g}+\sqrt{\frac{3}{8}}|2,2\rangle_{g}\right\}, \\
& \mathcal{H}_{g, 2}^{\text {inv }}=\operatorname{span}\left\{\frac{1}{\sqrt{2}}|2,-2\rangle_{g}-\frac{1}{\sqrt{2}}|2,2\rangle_{g}\right\}, \\
& \mathcal{H}_{g, 3}^{\text {inv }}=\operatorname{span}\left\{\frac{1}{\sqrt{2}}|2,-1\rangle_{g}-\frac{1}{\sqrt{2}}|2,1\rangle_{g}\right\}, \\
& \mathcal{H}_{g, 4}^{\text {inv }}=\operatorname{span}\left\{\frac{1}{2 \sqrt{2}}|2,-2\rangle_{g}+\frac{\sqrt{3}}{2}|2,0\rangle_{g}+\frac{1}{2 \sqrt{2}}|2,2\rangle_{g}\right\}, \\
& \mathcal{H}_{g, 5}^{\text {inv }}=\operatorname{span}\left\{\frac{1}{\sqrt{2}}|2,-1\rangle_{g}+\frac{1}{\sqrt{2}}|2,1\rangle_{g}\right\},
\end{aligned}
$$

and three invariant subspaces for $\boldsymbol{V}_{f} \boldsymbol{V}_{f}^{\dagger}$ and $\boldsymbol{S}_{f}$ :

$$
\begin{aligned}
& \mathcal{H}_{f, 1}^{\text {inv }}=\operatorname{span}\left\{|1,0\rangle_{f}\right\}, \\
& \mathcal{H}_{f, 2}^{\text {inv }}=\operatorname{span}\left\{\frac{1}{\sqrt{2}}|1,-1\rangle_{f}+\frac{1}{\sqrt{2}}|1,1\rangle_{f}\right\}, \\
& \mathcal{H}_{f, 3}^{\text {inv }}=\operatorname{span}\left\{\frac{1}{\sqrt{2}}|1,-1\rangle_{f}-\frac{1}{\sqrt{2}}|1,1\rangle_{f}\right\} .
\end{aligned}
$$

Six invariant subspaces of the Hamiltonian can be constructed using results (10)-(12):

$$
\begin{gathered}
\mathcal{H}_{1}^{\text {inv }}=\operatorname{span}\left\{\frac{1}{\sqrt{2}}|1,-1\rangle_{e}-\frac{1}{\sqrt{2}}|1,1\rangle_{e},\right. \\
\left.\sqrt{\frac{3}{8}}|2,-2\rangle_{g}-\frac{1}{2}|2,0\rangle_{g}+\sqrt{\frac{3}{8}}|2,2\rangle_{g}\right\}, \\
\mathcal{H}_{2}^{\text {inv }}=\operatorname{span}\left\{\frac{1}{\sqrt{2}}|1,-1\rangle_{e}+\frac{1}{\sqrt{2}}|1,1\rangle_{e},\right. \\
\left.\frac{1}{\sqrt{2}}|2,-2\rangle_{g}-\frac{1}{\sqrt{2}}|2,2\rangle_{g},|1,0\rangle_{f}\right\}, \\
\mathcal{H}_{3}^{\text {inv }}=\operatorname{span}\left\{|1,0\rangle_{e}, \frac{1}{\sqrt{2}}|2,-1\rangle_{g}-\frac{1}{\sqrt{2}}|2,1\rangle_{g},\right. \\
\left.\frac{1}{\sqrt{2}}|1,-1\rangle_{f}+\frac{1}{\sqrt{2}}|1,1\rangle_{f}\right\}, \\
\mathcal{H}_{4}^{\text {inv }}=\operatorname{span}\left\{\frac{1}{2 \sqrt{2}}|2,-2\rangle_{g}+\frac{\sqrt{3}}{2}|2,0\rangle_{g}\right. \\
\left.+\frac{1}{2 \sqrt{2}}|2,2\rangle_{g}\right\}, \\
\mathcal{H}_{5}^{\text {inv }}=\operatorname{span}\left\{\frac{1}{\sqrt{2}}|2,-1\rangle_{g}+\frac{1}{\sqrt{2}}|2,1\rangle_{g}\right\},
\end{gathered}
$$

$$
\mathcal{H}_{6}^{\mathrm{inv}}=\operatorname{span}\left\{\frac{1}{\sqrt{2}}|1,-1\rangle_{f}-\frac{1}{\sqrt{2}}|1,1\rangle_{f}\right\} .
$$

Subspaces $\mathcal{H}_{\kappa}^{\text {inv }}, \kappa \in\{2,3\}$, do not contain dark states, therefore it is possible to obtain complete population transfer from $\mathcal{H}_{g, \kappa^{\prime}}^{\text {inv }}$ to $\mathcal{H}_{f, \kappa^{\prime \prime}}^{\text {inv }}$ for pairs $\left(\kappa^{\prime}, \kappa^{\prime \prime}\right) \in$ $\{(2,1),(3,2)\}$. Let us note that the complete population transfer requires the starting states to be particular coherent superpositions. Conversely, the subspace $\mathcal{H}_{1}^{\text {inv }}$ contains the dark state from $\mathcal{H}_{e f}^{\mathrm{d}}$, so that the population transfers exclusively to the excited level, not to the final. The subspaces $\mathcal{H}_{f, 4}^{\text {inv }}$ and $\mathcal{H}_{f, 5}^{\text {inv }}\left(\mathcal{H}_{f, 6}^{\text {inv }}\right)$ are dark for transition from ground (final) to excited level and retain the initial population during the SCRAP process.

\section{Conclusion}

The analysis of a degenerate-level system is performed by its subdivision into a set of smaller independently evolving subsystems i.e. by finding the invariant subspaces of the Hamiltonian. Classification of the invariant subspaces is done depending on the presence of dark states. Amount of population transfer to final level is associated to each class of invariant subspaces. We examined SCRAP among three hyperfine levels in the rubidium atom. The presented description of the SCRAP population transfer process should be useful for analyzing adiabatic passage in a wide variety of atomic and molecular systems.

\section{Acknowledgments}

This work is supported by the Serbian Ministry of Science under grant No. 141003.

\section{References}

[1] L.P. Yatsenko, B.W. Shore, T. Halfmann, K. Bergmann, A. Vardi, Phys. Rev. A 60, R4237 (1999).

[2] T. Rickes, L.P. Yatsenko, S. Steuerwald, T. Halfmann, B.W. Shore, N.V. Vitanov, K. Bergmann, J. Chem. Phys. 113, 534 (2000).

[3] A.A. Rangelov, N.V. Vitanov, L.P. Yatsenko, B.W. Shore, T. Halfmann, K. Bergmann, Phys. Rev. A 72, 053403 (2005).

[4] M. Oberst, H. Münch, T. Halfmann, Phys. Rev. Lett. 99, 173001 (2007).

[5] M. Oberst, H. Müunch, G. Grigoryan, T. Halfmann, Phys. Rev. A 78, 033409 (2008).

[6] M. Radonjić, B.M. Jelenković, accepted in Phys. Rev. A. 